\title{
Celiac Disease and Autoimmunity: Review and Controversies
}

\author{
Jolanda M. Denham • Ivor D. Hill
}

Published online: 17 May 2013

(C) The Author(s) 2013. This article is published with open access at Springerlink.com

\begin{abstract}
Celiac disease (CD) is an autoimmune condition affecting the small intestine, triggered by the ingestion of gluten, the protein fraction of wheat, barley, and rye. There is a strong linkage between CD and HLA-DQ2 and HLADQ8 haplotypes. Multiple case reports and small series suggest concordance between $\mathrm{CD}$ and other autoimmune disorders. This paper provides a brief overview of the pathogenesis of $\mathrm{CD}$ and reviews the literature regarding associations between $\mathrm{CD}$ and other autoimmune diseases, including the potential effects of gluten-free diet therapy on the prevention or amelioration of associated diseases.
\end{abstract}

Keywords Celiac Celiac disease $\cdot$ Autoimmune $\cdot$

Addison's disease · Addison's · Type 1 diabetes ·

Autoimmune thyroiditis · Hashimoto's thyroiditis · Graves disease $\cdot$ Cryptogenic hepatitis $\cdot$ Celiac hepatitis $\cdot$ Primary biliary cirrhosis · Sjogren's syndrome $\cdot$ Systemic lupus erythematous · SLE · Lupus · Antiphospholipid syndrome · Psoriasis $\cdot$ Vitiligo $\cdot$ Alopecia acreta $\cdot$ Dermatitis herpetiformis

$\begin{array}{ll}\text { Abbreviations } \\ \text { AIH } & \text { Autoimmune hepatitis } \\ \text { APS } & \text { Antiphospholipid syndrome } \\ \text { AT } & \text { Autoimmune (Hashimoto's) thyroiditis } \\ \text { CD } & \text { Celiac disease } \\ \text { DH } & \text { Dermatitis herpetiformis } \\ \text { EMA } & \text { Endomysial antibody } \\ \text { eTG } & \text { Epidermal transglutaminase } \\ \text { GFD } & \text { Gluten-free diet } \\ \text { T1DM } & \text { Type 1 diabetes } \\ \text { tTG } & \text { Tissue transglutaminase }\end{array}$

J. M. Denham $(\varangle) \cdot$ I. D. Hill

Nationwide Children's Hospital, The Ohio State University School of Medicine, 700 Children's Drive,

Columbus, OH 43205, USA

e-mail: Jolanda.denham@nationwidechildrens.org

\section{Introduction}

Celiac disease (CD) is one of the most common chronic conditions affecting mankind. It has been identified throughout the world with the exception of SubSaharan Africa and East Asia. The prevalence of CD is usually reported to be about $1 \%$ in the general population, but there are emerging data to suggest it may actually be increasing in some developed countries. Clinical manifestations of CD are highly variable and include both gastrointestinal and non-gastrointestinal features. There are also people without any symptoms that have positive $\mathrm{CD}$ serology and characteristic histologic changes on small intestinal biopsy. These have been identified predominantly through screening groups who are at increased risk for CD. Others have symptoms and positive serological tests but do not initially have the characteristic histological features of mucosal damage. If these people remain on a regular diet, most will eventually go on to develop the mucosal changes found in $\mathrm{CD}$ over time. Because of these variations in clinical and laboratory findings, the precise definition of CD has resulted in much debate. Most recently, the European Society for Pediatric Gastroenterology, Hepatology and Nutrition proposed that $\mathrm{CD}$ be defined as follows, "... an immune-mediated systemic disorder elicited by gluten and related prolamines in genetically susceptible individuals and characterized by a variable combination of gluten-dependent manifestations, CD-specific antibodies, HLA-DQ2 or HLA-DQ8 haplotypes, and enteropathy" $[1 \bullet \bullet]$.

\section{Pathogenesis of CD}

As alluded to in the definition, $\mathrm{CD}$ is considered an autoimmune disorder with both a genetic and environmental component. Evidence for a genetic component is best exemplified by the strong dependence on the presence of the HLA-DQ2 (encoded by the alleles DQA1*05 and 
DQB1*02) and HLA-DQ8 (DQA1*03 and DQB1-*0302) haplotypes. More than $95 \%$ of those with CD express HLADQ2 while the remainder expresses HLA-DQ8. However, about $30-40 \%$ of the general population expresses HLADQ2, so while these HLA genes are necessary, they are not sufficient for developing CD and clearly non HLA genes are also involved. To date, at least 39 non-HLA genes have been identified through genome-wide association studies as strongly associated with CD [2]. Most of these genes are involved in control of the innate and adaptive immune response. However, individually each of these non-HLA genes is believed to play a relatively small role in CD.

The major environmental trigger is ingestion of "gluten", which is the term used to describe the protein fraction of wheat, barley, and rye. These proteins have high concentrations of glutamine and proline rendering them impossible to be completely digested by humans. It is these residual partially digested peptides that initiate innate and adaptive immune responses in those genetically predisposed to $\mathrm{CD}$. Other trigger factors may play a role in precipitating disease and could possibly account for the marked variability in age of onset and clinical manifestations of disease. Currently, there is interest in the role of the gut microbiome as an additional trigger factor contributing to the onset of disease.

\section{Immunopathogenesis of CD}

The hallmark of $\mathrm{CD}$ is an immune-mediated enteropathy that involves both the innate and adaptive immune system. Although the precise immune mechanisms that are involved in the progressive destruction of the small intestinal mucosa remain to be elucidated, some of the sequences have been identified. Initially gut paracellular permeability is increased in $\mathrm{CD}$ in part due to peptide-induced CXCR3 activated upregulation of zonulin, an intestinal peptide involved in epithelial tight junction control [3]. Paracellular passage of gliadin peptides follows. One peptide ( $\alpha$-gliadin $31-43$ ) has been shown to induce apoptosis of enterocytes, upregulate MHC class I molecules, activate MAP kinase pathway, and upregulate expression of CD83, a maturation marker of dendritic cells $[4,5]$. This peptide, and others, enhances IL15 production leading to an expansion of intraepithelial lymphocytes (IELs) and triggering the innate immune system. IL15 plays a key role in enhanced cytolytic activity of IELs via induction of NK receptors on the IEL and also contributes to promoting the CD4+ $\mathrm{T}$ cell adaptive response [6].

The adaptive immune response in $\mathrm{CD}$ is characterized predominantly by a gluten-specific $\mathrm{T}$ helper-1 response leading to production of the pro-inflammatory cytokine interferon- $\gamma$ (IFN- $\gamma)$ [7]. Tissue transglutaminase (TTG), now known to be the autoantigen in $\mathrm{CD}$, plays a key role in this process. By means of deamidation, TTG converts glutamine to glutamic acid at key sites within the gliadin peptide. This increases the negative charge on the peptide molecule and enhances binding of the peptide within the peptide binding groove of the HLA-DQ2 molecule on the surface of the antigen-presenting cells. This is a prerequisite for a gluten-specific $\mathrm{T}$ cell response as well as a B cell response that results in production of anti-TTG antibodies [3]. While these antibodies serve as a highly useful means of testing for $\mathrm{CD}$, their precise role in the immunopathogenesis of the condition remains unknown.

While much has been learned about the immune events that occur in CD, there is still more that is not known. There is evidence to support the role of IL21 in the pathogenesis of $\mathrm{CD}$ as high levels of this cytokine can be demonstrated in biopsies from those with active disease that are not on treatment [8]. However, the mechanism whereby IL21 is produced and the precise role it plays in the disease process remains unexplained. Perhaps of more importance is the need to better understand the interplay between the innate and adaptive immune system responses and how this leads to the villous atrophy that is characteristic of CD. Unraveling the mysteries of CD could offer major insights into other autoimmune diseases.

\section{CD and Other Autoimmune Diseases}

The tendency for multiple autoimmune disorders to occur over the lifetime of a patient with $\mathrm{CD}$ has been well described. To date, only hypotheses exist to explain the concordance of separate autoimmune diseases in individuals. Elucidation of the mechanisms of multiple autoimmune conditions in the same individual will provide deeper understanding of the specific conditions themselves.

\section{Celiac Disease and Type 1 Diabetes Mellitus}

The association between $\mathrm{CD}$ and type 1 diabetes mellitus (T1DM) is well established [9-13]. A recent review of the literature reported the prevalence of $\mathrm{CD}$ amongst adult and pediatric T1DM patients between 4.4 and $11 \%$ [14]. A large Swedish multicenter pediatric study of T1DM patients $(n=$ 300) screened yearly for celiac disease, reported that, over a 5 -year period, $6 \%$ of the cohort developed silent CD [13]. Other studies have shown that, in the vast majority ( $90 \%)$ of cases, the T1DM diagnosis precedes the celiac diagnosis $[15,16]$. The pathophysiology of the relationship between T1DM and celiac disease is not fully clear. Genetic studies show that celiac and T1DM share similar HLA and nonHLA genetic loci [9], suggesting the existence of autoimmunity genes. Treatment with a GFD in people with T1DM and $\mathrm{CD}$ is clearly beneficial in those who have symptoms 
associated with CD. Conversely, conflicting evidence exists as to whether a GFD significantly improves glycemic control in such cases [12, 16, 17].

\section{Celiac Disease and Addison's Disease}

The majority of Addison's disease is caused by autoimmune destruction of the adrenal cortex resulting in adrenal insufficiency [18, 19]. Studies show an association with HLADQ2 and HLA-DQ8, the same HLA haplotypes necessary for the presence of CD $[18,20]$. Small case series throughout the world report variable prevalence of $\mathrm{CD}$ amongst patients with Addison's disease and vice versa [20-24]. Elfstrom, et al. [25] reported the largest study of the occurrence of Addison's disease within a cohort of CD patients. In their series of adult Swedes, celiac patients were 11.4 times more likely to develop Addison's disease before or after their celiac diagnosis compared to non-celiac controls (95\% confidence interval $=4.4-29.6$ ).

\section{Celiac Disease and Thyroid Disorders}

The association between $\mathrm{CD}$ and thyroid disorders is well documented. Many studies confirm an increased prevalence of autoimmune (Hashimoto's) thyroiditis and Graves disease within adult [26-31] and pediatric [28, 32]celiac populations. The converse also appears to be true CD is found in persons with autoimmune thyroid disorders at higher rates than the general population [31,33-35]. As in the case of T1DM, HLA-DQ2 and DQ8 are disproportionately represented in patients with autoimmune thyroiditis [36] and Graves disease [37]. In their series of 90 pediatric patients with thyroid disorders, Larizza et al. reported a higher prevalence of autoimmune thyroiditis with $\mathrm{CD}$ compared to patients with isolated autoimmune thyroiditis in the general population. They also found $\mathrm{CD}$ to be the most common associated autoimmune disorder. CD occurred more frequently with autoimmune thyroiditis (6 of 68) compared to Graves disease (1 of 22); however, the comparison was statistically insignificant [38].

\section{Celiac Disease and Liver Disorders}

Cryptogenic hepatitis is the most common liver manifestation of $\mathrm{CD}$, followed by autoimmune hepatitis (AIH) [39-41]. Cryptogenic hepatitis has been typified by mild isolated transaminases with mild lobular and portal inflammation on liver biopsy (celiac hepatitis) [42]. In a large cohort of 350 pediatric celiac patients, Di Biase et al. reported isolated transaminase elevations in $40 \%$ of patients. Adult $[41,43,44]$ and pediatric $[45,46]$ studies have shown biochemical and histologic reversal of cryptogenic hepatitis within 1 year of gluten-free diet. An increased prevalence of AIH has been documented in both pediatric and adult $\mathrm{CD}$ cohorts, ranging from 2 to $11.5 \%$ $[39,45-50]$. CD has also been found at higher rates in AIH compared to non-autoimmune hepatitis populations [45, 51]. It is important to note that the HLA-DQ2 haplotype found in $95 \%$ of celiac patients shares a strong linkage to the HLA-DR3 haplotype associated with AIH [52]. Primary biliary cirrhosis (PBC) and primary sclerosing cholangitis (PSC) are rarely found in pediatric celiac patients, with rates between 0.005 and $0.04 \%$ [47, 53]. Prevalence rates for PBC and PSC are higher in adult studies. Amongst the South Wales adult population, Kingham et al. [54] reported a relative prevalence of $3 \%$ for $\mathrm{PBC}$ in 143 patients with $\mathrm{CD}$ and, amongst 67 patients, a relative prevalence of $6 \%$ for PSC. Within a series of 255 adult autoimmune cholestasis patients, $3.5 \%$ had $\mathrm{CD}$ (7 primary biliary cirrhosis, 1 primary sclerosing cholangitis and 1 autoimmune cholangitis) [55]. The pathophysiology of the association between autoimmune hepatitis and $\mathrm{CD}$ remains unclear. Volta et al. [40] proposed increased intestinal permeability and circulation of anti-TTG antibodies modify external or self antigens. These modifications then generate neoantigens which, in turn, cause liver damage.

\section{Celiac Disease and Rheumatologic Disorders}

Sjogren's syndrome (SS) is an autoimmune exocrinopathy typified by dry eyes, dry mouth, and circulating antibodies to intracellular proteins [56]. An association between Sjogren's syndrome and CD was reported as early as 1965 [57]. Since that time, two small series have reported prevalence rates of CD amongst adult SS patients as $12 \%$ [58] and $14.4 \%$ [59]. With these values, Sjogren's syndrome is considered the most common rheumatic disorder associated with $C D$. Fifty-six percent of SS adult patients also had HLA-DQ2, the haplotype most commonly found in $\mathrm{CD}$ [58]. A number of case reports describe an association between systemic lupus erythematosus (SLE) and CD [60-63]. However, none of the participants in a large series of 103 SLE patients had CD (biopsy and EMA negative) [64]. The prevalence of $\mathrm{CD}$ amongst patients with chronic arthritis has been reported as $1.5 \%$ [65] and $2.5 \%$ [66].

Antiphospholipid syndrome (APS) is disorder characterized by clinical (vascular thrombosis, and pregnancy complications) as well as laboratory (lupus anticoagulant, anticardiolipin) criteria [67]. Shamir et al. [68] reported a CD prevalence of $14 \%$ amongs 57 adult with APS. Conversely, $100 \%$ of the EMA positive 
patients had APS. Luft et al. reported CD prevalences in the following rheumatoid adult populations: $12 \%$ in SS, $6 \%$ in SLE, $7 \%$ in systemic sclerosis (SSc), and $2 \%$ in rheumatoid arthritis (RA) [58]. Given the frequency of hypergammaglobulinemia in these disorders, the authors expected a high occurence of false positive antiTTG. Interestingly, this was not observed.

\section{Celiac Disease and Dermatologic Disorders}

Dermatitis herpetiformis is the dermatologic manifestation of CD [69•]. The rash typically involves the elbows, extensor surfaces of the forearms, knees, buttocks, back, and scalp. Lesions begin as groups of 1- to 3-mm papules and vesicles, but due to intense pruritis, the lesions become the crusted erosions, excoriations and lichenified tissue that is typical of the rash visualized by medical personnel. Often, the pruritis precedes the onset of rash. Lesions can present at any age, but occur most commonly in young to middle aged adults $[69 \bullet, 70,71]$. The rash is chronic, with exacerbations usually associated with sweating, alternating with long symptom-free periods. DH in pediatric patients presents as urticaria, and more commonly purpura involving the soles, palms, and sides of the fingers. Dermal biopsies of lesions and surrounding tissues show epidermal tissue transglutaminase (eTG), the autoantigen for $\mathrm{DH}$, complexed to immunoglobin A. These complexes trigger an immune cascade analogous to the tTG-IgA immune cascade in the gastrointestinal tract $[72,73]$. Despite DH spontaneously resolving in up to $12 \%$ of patients [74], GFD is the standard treatment for $\mathrm{DH}$, with resolution in $100 \%$ patients $[69 \bullet, 71]$. Dapsone, $50-100 \mathrm{mg} /$ day, rapidly ameliorates the rash, but does not halt the intestinal damage caused by ongoing gluten exposure. Idiosyndratic agranulocytosis can occur rarely with dapsone usage [71]. Concomittant administration of cimetidine $400 \mathrm{mg}$ TID [75], or vitamin $\mathrm{C}$ can prevent dapsoneinduced methemoglobinemia [69•]. Oral steroids do not help $\mathrm{DH}$, but topical steroid creams can be used as adjuncts to dapsone [71].

Psoriasis is a $\mathrm{T}$ cell-mediated autoimmune disorder which causes skin erythema, scaling, and occassionally arthritis [76]. Its association with CD remains unclear. Some small studies support an association [77-79], while others show no relationship [80, 81]. A very large, population-based Swedish study of almost 28,958 adults and children with $\mathrm{CD}$ showed a 1.72 hazzard ratio for lifetime psoriasis development amongst adults and 2.05 amongst children [82]. The psoriasis could precede or follow the celiac diagnosis. A number of hypotheses regarding the association of psoriasis and CD exist: (1) the vitamin D deficiency commonly seen in $\mathrm{CD}$ [83] is known to predispose patients to development of psoriasis $[84,85],(2)$ the $\mathrm{T}$ cell proliferation and activity in intestinal mucosa may extend into the blood stream, eventually reaching the dermis and epidermis, the location of the psoriatic immune process [86-88], and (3) psoriasis is known to increase intestinal permeability [89] which is one of the first steps in the pathogenesis of CD [90].

A few case reports suggest an association between $\mathrm{CD}$ and alopecia acreta (AA) $[91,92]$. In a prospective trial of 256 AA patients, 6 had positive TTG and EMA with subsequent biopsy confirmation [93]. Seyhan et al. [94] reported a prevalence of $9 \%$ for vitiligo amongst adult celiac patients. In a later study of 61 adult and pediatric patients with vitiligo, the same group reported prevalences rates of $15 \%$ for $\mathrm{CD}$ amongst adult vitiligo patients, and $24 \%$ amongst the pediatric cohort [95].

\section{Effect of Gluten-Free Diet on Autoimmune Disorders}

Numerous papers have investigated the effect of CD treatment on the incidence and prognosis of various autoimmune disorders. The data are conflicting. Ventura et al. prospectively studied 90 adults with biopsy-proven $\mathrm{CD}$ to determine the levels of autoimmune antibodies associated with type 1 diabetes (glutamic acid decarboxylase, islet cell, anti-insulin) and autoimmune thyroiditis (anti-thyroperoxidase), at celiac diagnosis, and then at intervals up to 2 years while on a GFD. They report a prevalence of type 1 diabetes -related and anti-thyroid related antibodies of 11.1 and $14.4 \%$, respectively. Within 2 years of avoiding gluten, all antibodies normalized. The authors, therefore, surmised that a GFD was therapeutic against related autoimmunity [96]. In another large series of 927 adult and pediatric French celiac patients, Cosnes et al. reported the incidence of autoimmune diseases to be lower in the compliant gluten-free diet group compared to the non-compliant group $(5.4 / 1,000$ vs. $11.3 / 1,000$ patient-years during $(P=.002))$ [29]. Intriguingly, the authors also showed that patients first diagnosed with $\mathrm{CD}$ when older than 36 years had a decreased cumulative risk of autoimmune disorders compared to those diagnosed between 16 and 36 or younger than 16 years old. They proposed that given the premise that $\mathrm{CD}$ is a manifestation of autoimmune dysregulation, older celiac patients are less susceptible to autoimmunity, $\mathrm{CD}$, or otherwise. In addition, with later celiac onset, the integrity of the intestinal barrier has been more competent, reducing the antigen triggers for various autoimmune disorders [29]. This is in contrast to the findings of Ventura et al. who studied 909 patients with $\mathrm{CD}$ and concluded that 
increased duration of exposure to gluten was related to a higher prevalence of other autoimmune disorders [47].

Evidence against the protective effect of a GFD exists. In a prospective study of 27 adult biopsy-proved celiac patients, Metso et al. found celiac patients had an elevated anti-TPO, which continued to increase despite nutrition-guided compliance with a GFD. Thyroid atrophy also progressed while on a GFD. The authors suggest that autoimmune diseases can be reverted in childhood, but not in adulthood [97]. Valentino et al. reported improvement of hypothyroidism and reduction of the thyroxine dosage with a GFD in 5 patients with both CD and autoimmune thyroiditis [98]. Conversely, in Lirazza et al's series of 52 celiac pediatric patients treated with L-thyroxine for autoimmune thyroiditis, 51 exhibited no difference in their thyroperoxidase antibody levels or reduction in their Lthyroxine levels, while 1 patient was able to wean off autoimmune thyroiditis therapy within 5 years [38]. Other studies report no difference in autoimmune thyroiditis with a GFD $[30,32,97,99]$.

In a large cohort of pediatric patients with T1DM, no difference was observed in hemoglobin A1C (HbA1C) levels, frequency of ketoacidosis, hypoglycemia, or need for increased insulin dosing between celiac patients and controls [100]. In a large investigation of arthritis among 356 adult celiac patients, $49 \%$ reported "some" improvement with a GFD, and $30 \%$ attributed the improvement to the GFD [101].

\section{Conclusions}

$\mathrm{CD}$ has been associated with various autoimmune disorders, but there are no good data to establish a cause and effect relationship between $\mathrm{CD}$, gluten, and these autoimmune conditions. Current evidence is derived largely from case reports and small case series, and there is a paucity of large, population-based prospective studies necessary to clarify this issue. Review of the literature shows that $\mathrm{CD}$ is most strongly associated with autoimmune thyroiditis, autoimmune hepatitis, type 1 diabetes, DH, Sjogren's syndrome, and psoriasis. Given these specific associations, the propensity toward multiple autoimmune diseases in any one individual, and the variable presentations of $\mathrm{CD}$, one should have a low threshold for screening affected patients with TTG and/or EMA serology. Current recommendations are that the diagnosis of $\mathrm{CD}$ be confirmed by means of intestinal histology in all cases prior to starting treatment with a GFD. Additional large prospective studies are needed to clarify the relationship between CD and other autoimmune disorders and the potential effect of the GFD on these conditions. Further delineation of these relationships will add to deeper understanding of the individual diseases and to autoimmunity in general.
Conflict of Interest Jolanda M. Denham declares that she has no conflict of interest.

Ivor D. Hill declares that he has no conflict of interest.

Open Access This article is distributed under the terms of the Creative Commons Attribution License which permits any use, distribution, and reproduction in any medium, provided the original author(s) and the source are credited.

\section{References}

Papers of particular interest, published recently, have been highlighted as:

- Of importance

-. Of major importance

1. • Husby S et al. European Society for Pediatric Gastroenterology, Hepatology, and Nutrition guidelines for the diagnosis of coeliac disease. J Pediatr Gastroenterol Nutr. 2012;54(1):13660. Celiac position statement for pediatric celiac disease.

2. Kumar V, Wijmenga C, Withoff S. From genome-wide association studies to disease mechanisms: celiac disease as a model for autoimmune diseases. Semin Immunopathol. 2012;34(4):567-80.

3. Lammers $\mathrm{KM}$ et al. Gliadin induces an increase in intestinal permeability and zonulin release by binding to the chemokine receptor CXCR3. Gastroenterology. 2008;135(1):194204 e3.

4. Maiuri $\mathrm{L}$ et al. Association between innate response to gliadin and activation of pathogenic $\mathrm{T}$ cells in coeliac disease. Lancet. 2003;362(9377):30-7.

5. Hue $\mathrm{S}$ et al. A direct role for NKG2D/MICA interaction in villous atrophy during celiac disease. Immunity. 2004;21(3):367-77.

6. Tang F et al. Cytosolic PLA2 is required for CTL-mediated immunopathology of celiac disease via NKG2D and IL-15. J Exp Med. 2009;206(3):707-19.

7. Nilsen EM et al. Gluten specific, HLA-DQ restricted T cells from coeliac mucosa produce cytokines with Th1 or Th0 profile dominated by interferon gamma. Gut. 1995;37(6):766-76.

8. Fina $\mathrm{D}$ et al. Interleukin 21 contributes to the mucosal $\mathrm{T}$ helper cell type 1 response in coeliac disease. Gut. 2008; 57(7):887-92.

9. Smyth DJ et al. Shared and distinct genetic variants in type 1 diabetes and celiac disease. N Engl J Med. 2008;359(26):2767-77.

10. Sanchez-Albisua I et al. Coeliac disease in children with Type 1 diabetes mellitus: the effect of the gluten-free diet. Diabet Med. 2005;22(8):1079-82.

11. Araujo J, da Silva GA, de Melo FM. Serum prevalence of celiac disease in children and adolescents with type 1 diabetes mellitus. J Pediatr (Rio J). 2006;82(3):210-4.

12. Goh C, Banerjee K. Prevalence of coeliac disease in children and adolescents with type 1 diabetes mellitus in a clinic based population. Postgrad Med J. 2007;83(976):132-6.

13. Larsson $\mathrm{K}$ et al. Annual screening detects celiac disease in children with type 1 diabetes. Pediatr Diabetes. 2008;9(4 Pt 2):354-9.

14. Camarca ME et al. Celiac disease in type 1 diabetes mellitus. Ital J Pediatr. 2012;38:10.

15. Pocecco M, Ventura A. Coeliac disease and insulin-dependent diabetes mellitus: a causal association? Acta Paediatr. 1995;84(12):1432-3.

16. Kaspers $\mathrm{S}$ et al. Anthropometry, metabolic control, and thyroid autoimmunity in type 1 diabetes with celiac disease: a multicenter survey. J Pediatr. 2004;145(6):790-5. 
17. Kaukinen $\mathrm{K}$ et al. No effect of gluten-free diet on the metabolic control of type 1 diabetes in patients with diabetes and celiac disease. Retrospective and controlled prospective survey. Diabetes Care. 1999;22(10):1747-8.

18. Myhre AG et al. Autoimmune adrenocortical failure in Norway autoantibodies and human leukocyte antigen class II associations related to clinical features. J Clin Endocrinol Metab. 2002;87(2):618-23.

19. Zelissen PM, Bast EJ, Croughs RJ. Associated autoimmunity in Addison's disease. J Autoimmun. 1995;8(1):121-30.

20. O'Leary $\mathrm{C}$ et al. Coeliac disease and autoimmune Addison's disease: a clinical pitfall. QJM. 2002;95(2):79-82.

21. Biagi $\mathrm{F}$ et al. Prevalence of coeliac disease in Italian patients affected by Addison's disease. Scand J Gastroenterol. 2006;41(3):302-5.

22. Betterle $\mathrm{C}$ et al. Celiac disease in North Italian patients with autoimmune Addison's disease. Eur J Endocrinol. 2006;154(2):275-9.

23. Kaukinen $\mathrm{K}$ et al. Celiac disease and autoimmune endocrinologic disorders. Dig Dis Sci. 1999;44(7):1428-33.

24. Myhre AG et al. High frequency of coeliac disease among patients with autoimmune adrenocortical failure. Scand J Gastroenterol. 2003;38(5):511-5.

25. Elfstrom $P$ et al. Risk of primary adrenal insufficiency in patients with celiac disease. J Clin Endocrinol Metab. 2007;92(9):3595-8.

26. Sategna-Guidetti $C$ et al. Prevalence of thyroid disorders in untreated adult celiac disease patients and effect of gluten withdrawal: an Italian multicenter study. Am J Gastroenterol. 2001;96(3):751-7.

27. Sategna Guidetti $C$ et al. Duration of gluten exposure in adult coeliac disease does not correlate with the risk for autoimmune disorders. Gut. 2001;49(4):502-5.

28. Collin $\mathrm{P}$ et al. Endocrinological disorders and celiac disease. Endocr Rev. 2002;23(4):464-83.

29. Cosnes $J$ et al. Incidence of autoimmune diseases in celiac disease: protective effect of the gluten-free diet. Clin Gastroenterol Hepatol. 2008;6(7):753-8.

30. Viljamaa $\mathrm{M}$ et al. Coeliac disease, autoimmune diseases and gluten exposure. Scand J Gastroenterol. 2005;40(4):437-43.

31. Hadithi $M$ et al. Coeliac disease in Dutch patients with Hashimoto's thyroiditis and vice versa. World J Gastroenterol. 2007;13(11):1715-22.

32. Meloni A et al. Prevalence of autoimmune thyroiditis in children with celiac disease and effect of gluten withdrawal. J Pediatr. 2009;155(1):51-5, 55 e1.

33. Spadaccino AC et al. Celiac disease in North Italian patients with autoimmune thyroid diseases. Autoimmunity. 2008;41(1):116-21.

34. Boelaert $\mathrm{K}$ et al. Prevalence and relative risk of other autoimmune diseases in subjects with autoimmune thyroid disease. Am J Med. 2010;123(2):183 e1-9.

35. Volta $U$ et al. Coeliac disease in patients with autoimmune thyroiditis. Digestion. 2001;64(1):61-5.

36. Dayan CM, Daniels GH. Chronic autoimmune thyroiditis. N Engl J Med. 1996;335(2):99-107.

37. Ch'ng CL et al. Prospective screening for coeliac disease in patients with Graves' hyperthyroidism using anti-gliadin and tissue transglutaminase antibodies. Clin Endocrinol (Oxf). 2005;62(3):303-6.

38. Larizza D et al. Celiac disease in children with autoimmune thyroid disease. J Pediatr. 2001;139(5):738-40

39. Rubio-Tapia A, Murray J. The liver in celiac disease. Hepatology. 2007;46:1650-8.

40. Volta U. Pathogenesis and clinical significance of liver injury in celiac disease. Clin Rev Allergy Immunol. 2009;36(1):62-70.

41. Duggan JM, Duggan AE. Systematic review: the liver in coeliac disease. Aliment Pharmacol Ther. 2005;21(5):515-8.
42. Hagander B et al. Hepatic injury in adult coeliac disease. Lancet. 1977;2(8032):270-2.

43. Novacek G et al. Prevalence and clinical importance of hypertransaminasaemia in coeliac disease. Eur $\mathrm{J}$ Gastroenterol Hepatol. 1999;11(3):283-8.

44. Bardella MT et al. Prevalence of hypertransaminasemia in adult celiac patients and effect of gluten-free diet. Hepatology. 1995;22(3):833-6.

45. Caprai $\mathrm{S}$ et al. Autoimmune liver disease associated with celiac disease in childhood: a multicenter study. Clin Gastroenterol Hepatol. 2008;6(7):803-6.

46. Di Biase AR, et al. Autoimmune liver diseases in a paediatric population with coeliac disease - a 10-year single-centre experience. In Aliment Pharmacol Ther; 2010, p. 253-60.

47. Ventura A, Magazzu G, Greco L. Duration of exposure to gluten and risk for autoimmune disorders in patients with celiac disease. SIGEP Study Group for Autoimmune Disorders in Celiac Disease. Gastroenterology. 1999;117(2):297-303.

48. Diamanti A et al. Prevalence of celiac disease in children with autoimmune hepatitis. Dig Liver Dis. 2008;40(12):965.

49. El-Shabrawi $\mathrm{M}$ et al. Celiac disease in children and adolescents with autoimmune hepatitis: a single-centre experience. J Trop Pediatr. 2011;57(2):104-8.

50. Tosun MS, Ertekin V, Selimoglu MA. Autoimmune hepatitis associated with celiac disease in childhood. Eur J Gastroenterol Hepatol. 2010;22(7):898-9.

51. Mirzaagha $\mathrm{F}$ et al. Coeliac disease in autoimmune liver disease: a cross-sectional study and a systematic review. Dig Liver Dis. 2010;42(9):620-3.

52. Czaja AJ, Doherty DG, Donaldson PT. Genetic bases of autoimmune hepatitis. Dig Dis Sci. 2002;47(10):2139-50.

53. Ludvigsson JF et al. Celiac disease and risk of liver disease: a general population-based study. Clin Gastroenterol Hepatol. 2007;5(1):63-69 e1.

54. Kingham JG, Parker DR. The association between primary biliary cirrhosis and coeliac disease: a study of relative prevalences. Gut. 1998;42(1):120-2.

55. Volta $U$ et al. Celiac disease in autoimmune cholestatic liver disorders. Am J Gastroenterol. 2002;97(10):2609-13.

56. Fox RI, Chan EK, Kang HI. Laboratory evaluation of patients with Sjogren's syndrome. Clin Biochem. 1992;25(3):213-22.

57. Pittman FE, Holub DA. Sjoegren's syndrome and adult celiac disease. Gastroenterology. 1965;48:869-76.

58. Luft LM et al. Autoantibodies to tissue transglutaminase in Sjogren's syndrome and related rheumatic diseases. J Rheumatol. 2003;30(12):2613-9.

59. Iltanen $\mathrm{S}$ et al. Celiac disease and markers of celiac disease latency in patients with primary Sjogren's syndrome. Am J Gastroenterol. 1999;94(4):1042-6.

60. Komatireddy GR et al. Association of systemic lupus erythematosus and gluten enteropathy. South Med J. 1995;88(6):673-6.

61. Mukamel $\mathrm{M}$ et al. Celiac disease associated with systemic lupus erythematosus. Isr J Med Sci. 1994;30(8):656-8.

62. Rustgi AK, Peppercorn MA. Gluten-sensitive enteropathy and systemic lupus erythematosus. Arch Intern Med. 1988;148(7):1583-4.

63. Pena AS. Systemic lupus erythematosus, Sjogren's syndrome, and purpura in a patient with coeliac disease. Neth $\mathrm{J}$ Med. 1987;31(5-6):305-7.

64. Rensch MJ et al. The prevalence of celiac disease autoantibodies in patients with systemic lupus erythematosus. Am J Gastroenterol. 2001;96(4):1113-5.

65. George EK et al. Juvenile chronic arthritis and coeliac disease in The Netherlands. Clin Exp Rheumatol. 1996;14(5):571-5.

66. Lepore L et al. Prevalence of celiac disease in patients with juvenile chronic arthritis. J Pediatr. 1996;129(2):311-3. 
67. Levine JS, Branch DW, Rauch J. The antiphospholipid syndrome. N Engl J Med. 2002;346(10):752-63.

68. Shamir R et al. The prevalence of coeliac disease antibodies in patients with the antiphospholipid syndrome. Lupus. 2003;12(5):394-9.

69. Karpati S. Dermatitis herpetiformis. Clin Dermatol. 2012;30(1):56-9. Comprehensive review of dermatitis herpetiformis.

70. Bolotin D, Petronic-Rosic V. Dermatitis herpetiformis. Part I. Epidemiology, pathogenesis, and clinical presentation. J Am Acad Dermatol. 2011;64(6):1017-24. quiz 1025-6.

71. Bolotin D, Petronic-Rosic V. Dermatitis herpetiformis. Part II. Diagnosis, management, and prognosis. J Am Acad Dermatol. 2011;64(6):1027-33. quiz 1033-4.

72. Sardy $M$ et al. Epidermal transglutaminase (TGase 3) is the autoantigen of dermatitis herpetiformis. J Exp Med. 2002;195(6):747-57.

73. Donaldson MR et al. Epidermal transglutaminase deposits in perilesional and uninvolved skin in patients with dermatitis herpetiformis. J Invest Dermatol. 2007;127(5):1268-71.

74. Paek SY, Steinberg SM, Katz SI. Remission in dermatitis herpetiformis: a cohort study. Arch Dermatol. 2011;147(3):301-5.

75. Coleman MD et al. The use of cimetidine to reduce dapsonedependent methaemoglobinaemia in dermatitis herpetiformis patients. Br J Clin Pharmacol. 1992;34(3):244-9.

76. Kirby B, Griffiths CE. Novel immune-based therapies for psoriasis. Br J Dermatol. 2002;146(4):546-51.

77. Ojetti V et al. High prevalence of celiac disease in psoriasis. Am J Gastroenterol. 2003;98(11):2574-5.

78. Birkenfeld S et al. Coeliac disease associated with psoriasis. Br J Dermatol. 2009;161(6):1331-4.

79. Singh S, Sonkar GK, Usha NO. Celiac disease-associated antibodies in patients with psoriasis and correlation with HLA Cw6. J Clin Lab Anal. 2010;24(4):269-72.

80. Collin P, Reunala T. Recognition and management of the cutaneous manifestations of celiac disease: a guide for dermatologists. Am J Clin Dermatol. 2003;4(1):13-20.

81. Kia KF et al. Prevalence of antigliadin antibodies in patients with psoriasis is not elevated compared with controls. Am J Clin Dermatol. 2007;8(5):301-5.

82. Ludvigsson JF et al. Psoriasis in a nationwide cohort study of patients with celiac disease. J Invest Dermatol. 2011;131(10):2010-6.

83. Cellier $\mathrm{C}$ et al. Severe osteopenia in symptom-free adults with a childhood diagnosis of coeliac disease. Lancet. 2000;355(9206):806.

84. Hein G et al. Studies on psoriatic osteopathy. Clin Rheumatol. 1991;10(1):13-7.
85. Holick MF. Vitamin D: a millenium perspective. J Cell Biochem. 2003;88(2):296-307.

86. Addolorato $\mathrm{G}$ et al. Rapid regression of psoriasis in a coeliac patient after gluten-free diet. A case report and review of the literature. Digestion. 2003;68(1):9-12.

87. Mailliard RB et al. Complementary dendritic cell-activating function of CD8+ and CD4+ T cells: helper role of CD8+ T cells in the development of $\mathrm{T}$ helper type 1 responses. J Exp Med. 2002;195(4):473-83.

88. Molberg $\mathrm{O}$ et al. Tissue transglutaminase selectively modifies gliadin peptides that are recognized by gut-derived $\mathrm{T}$ cells in celiac disease. Nat Med. 1998;4(6):713-7.

89. Humbert $P$ et al. Intestinal permeability in patients with psoriasis. J Dermatol Sci. 1991;2(4):324-6.

90. Montalto $\mathrm{M}$ et al. Immunohistochemical analysis of ZO-1 in the duodenal mucosa of patients with untreated and treated celiac disease. Digestion. 2002;65(4):227-33.

91. Fessatou S, Kostaki M, Karpathios T. Coeliac disease and alopecia areata in childhood. J Paediatr Child Health. 2003;39(2):152-4.

92. Naveh Y et al. Celiac disease-associated alopecia in childhood. J Pediatr. 1999;134(3):362-4.

93. Corazza GR et al. Celiac disease and alopecia areata: report of a new association. Gastroenterology. 1995;109(4):1333-7.

94. Seyhan $\mathrm{M}$ et al. The mucocutaneous manifestations associated with celiac disease in childhood and adolescence. Pediatr Dermatol. 2007;24(1):28-33.

95. Seyhan $\mathrm{M}$ et al. Is celiac disease common in patients with vitiligo? Turk J Gastroenterol. 2011;22(1):105-6.

96. Ventura A et al. Gluten-dependent diabetes-related and thyroidrelated autoantibodies in patients with celiac disease. J Pediatr. 2000;137(2):263-5.

97. Metso $\mathrm{S}$ et al. Gluten-free diet and autoimmune thyroiditis in patients with celiac disease. A prospective controlled study. Scand J Gastroenterol. 2012;47(1):43-8.

98. Valentino R et al. Prevalence of coeliac disease in patients with thyroid autoimmunity. Horm Res. 1999;51(3):124-7.

99. Cassio A et al. Long-term clinical significance of thyroid autoimmunity in children with celiac disease. J Pediatr. 2010;156(2):292-5.

100. Rami B et al. Screening detected celiac disease in children with type 1 diabetes mellitus: effect on the clinical course (a case control study). J Pediatr Gastroenterol Nutr. 2005;41(3):317-21.

101. Iqbal $\mathrm{T}$ et al. Celiac disease arthropathy and autoimmunity study. J Gastroenterol Hepatol. 2013;28(1):99-105. 